\title{
TYPOLOGISCHE AUFGABEN DER HISTORISCHEN TEXTLINGUISTIK
}

\section{Zum Verhältnis von Historiolinguistik und Textlinguistik}

Die gegenwärtige germanistische Historiolinguistik ist geprägt durch eine pragmatische Ausweitung ihres traditionell sprachstrukturellen Erkenntnisinteresses. Ausdruck dieser Entwicklung ist z.B. die gänzliche Neubearbeitung der Sprachgeschichte von P. von Polenz (1991 und 1994), die an Stelle der wiederholt neu aufgelegten und auf H. Sperber (1926) grüdenden Geschichte der deutschen Sprache (von Polenz 1978) erschienen ist und insbesondere eine "sozial- und mediengeschichtliche Fundierung" (ebd., 3) anstrebt, wobei neben anderen Aspekten als Schwerpunkt der Darstellung explizit die Sprachpragmatik genannt wird (ebd.). Sind die konzeptionellen Ecksteine einer solchen pragmatischen Sprachgeschichtsschreibung auch schon Anfang der 1980 er Jahre insbesondere mit H. Sitta (1980) und D. Cherubim (1984) gesetzt, so verstärkt sich das sprachhandlungsbezogene Interesse an der Geschichte des Deutschen erst in jüngster Zeit. Die Entwicklung läuft dabei parallel zur Konstituierung eines pragmatischen Paradigmas, das als Gemeinschaftswerk geisteswissenschaftlicher Forschung ebenfalls in den 1980er Jahren an Bedeutung gewann und als dessen notwendige Folge H. Stachowiak ([Hg.] 1986, XVII) einen "'Paradigmawechsel' großen Stils" prophezeit hat. ${ }^{1}$ Bei den Pragmatisierungstendenzen sprachgeschichtlicher Fragestellungen kommt der historischen Dimensionierung textlinguistischer Erkenntnisfelder besondere Bedeutung zu. Es ist bekannt, daß die in den späten 1960er und 70er Jahren begründete Textlinguistik zunächst ebenso ahistorisch gewesen ist, wie die Historiolinguistik apragmatisch. Doch mit der fortschreitenden Rezeption sprachhandlungsorientierter Ansätze in der Sprachgeschichte gewann der Text i.S. einer komplexen sprachlichen Handlung (vgl. u.a. Sandig 1978, 69f., 99ff., 157f.) als Analyseobjekt für die pragmatische Orientierung der Sprachgeschichte zunehmend an Bedeutung. In diesem Zusammenhang ist die Forderung nach textlinguistischer bzw. textsortenbe-

1 Für den interdisziplinären Aspekt der Pragmatik im Rahmen geisteswissenschaftlicher Forschung ist insbesondere die von $\mathrm{H}$. Stachowiak ([Hg.] 1986ff) herausgebene Handbuchreihe pragmatischen Denkens von Wichtigkeit, sowie der in das Projekt Pragmatik einführende Aufsatz von $\mathrm{H}$. Stachowiak (1986). 
zogener Fokussierung der Sprachgeschichtsschreibung vielfach formuliert worden. Als Beispiel sei hier aus H. Stegers $(1984,200)$ grundlegendem Aufsatz Sprachgeschichte als Geschichte der Textsorten/Texttypen ... zitiert:

"Zusammenfassend betrachtet stellt sich die deutsche Sprachgeschichte (auch) dar als Geschichte der Ausgliederung und des Ausbaus von funktionalen Sprachvarietäten und Texttypen-/Gattungsinventaren, ...".

Nun fällt auf, daß der Vielzahl programmatischer Forderungen nach einer Textorientierung sprachgeschichtlicher Fragestellungen nur wenige konkrete Textanalysen gegenüberstehen. Es scheint also, als sei zwar ein neues historiolinguistisches Paradigma eröffnet, das jedoch weder durch empirische Befunde ausreichend gedeckt noch in Frage gestellt ist. Es mag verschiedene Gründe für diesen Praxis-Theorie-Bruch der gegenwärtigen textorientierten Sprachgeschichtsschreibung geben; drei Aspekte sollen hier zunächst als Thesen formuliert werden:

(1) Die bisherigen sprachgeschichtlichen Forderungen nach einer Darstellung der Textsortengeschichte des Deutschen sind nicht hinreichend in den Zusammenhang übergreifender sprachhistorischer Problemstellungen gerückt. Historische Textlinguistik ist aber letzthin erst legitimiert, wenn sie über die Systematisierung von Quellen hinaus zur Beantwortung sprachhistorischer Fragestellungen beiträgt.

(2) Das Programm Sprachgeschichte als Textsortengeschichte ist bisher nicht in genügendem Maße mit der Konzeption eines pragmatisch orientierten Verfahrens zur Analyse historischer Texte verknüpft. Ausnahmen, wie J. Schwitallas (1983) Untersuchung zur Textsorte 'Flugschrift' gibt es zwar, doch haben sie noch nicht zu einer grundsätzlichen textlinguistischen Theorieorientierung pragmatischer Sprachgeschichtsschreibung beigetragen.

(3) Die bisherige Sprachgeschichtsschreibung des Deutschen hat noch nicht aufzeigen können, daß die Genese eines Textbereiches in unmittelbarem Zusammenhang mit sprachhistorischen Entwicklungsprozessen steht, so daß die traditionelle grammatische Erklärung von Sprachwandlungsvorgängen nicht grundsätzlich modifiziert bzw. in Frage gestellt ist.

Weitere Gründe für das Auseinanderfallen von programmatischen Forderungen und tatsächlichen textorientierten Untersuchungen in der germanistischen Sprachgeschichtsschreibung gibt es zweifellos - so mag etwa der erhebliche philologische Arbeitsaufwand bei einer systematischen Erschließung historischer Texttypeninventare schnelle Fortschritte im empirischen Bereich erschweren -, doch die nachfolgende Argumentation wird allein von den drei Thesen ausgehen und von hier aus konzeptionelle Vorschläge für historisch-pragmatische Textanalysen entfalten. Für die Thematik des vorliegenden Bandes ist eine Auseinandersetzung mit These (2), also mit dem Desiderat eines textlinguistischen Konzepts zur Analyse historischer Texte am wichtigsten, zumal damit nicht nur ein diachroner Problembereich anschaulich gemacht werden kann, sondern die prinzipiellen Fragestellungen zur Texttypologie und Textsortenklassifikation 
angesprochen sind. Die auf These (1) und (3) bezogenen Auseinandersetzungen werden mithin auf den nötigsten Umfang beschränkt. Außer acht können sie jedoch im Argumentationszusammenhang nicht gelassen werden, da ihre Behandlung Bedingung der Möglichkeit einer sinnvollen textbezogenen Historiolinguistik ist. Entsprechend soll zunächst auf die mit These (1) bezeichneten Probleme knapp eingegangen werden.

\section{Zum sprachgeschichtlichen Problembezug historischer Textlinguistik}

Textsortengeschichtliche Darstellungen der letzten Jahre haben bisher eher einen Beitrag zur Systematisierung des Quellenbestandes von raum-zeitlich definierten Sprachperioden geleistet, als eine Beschreibung der historischen Dynamik sprachlicher Handlungsverfahren. Exemplarisch ist dies an den textsortengeschichtlichen Beiträgen im sprachgeschichtlichen Handbuch von W. Besch/ O. Reichmann/ St. Sonderegger (1984/85) abzulesen. Obgleich das Handbuch angemessene Vollständigkeit "erstens im Hinblick auf den zu beschreibenden Gegenstand und zweitens im Hinblick auf die Forschungssituation" anstrebt (ebd., Bd. 1, IX), bleibt die Behandlung des Stellenwertes der Textsortengeschichte im Rahmen sprachhistorischer Leitfragen marginal. ${ }^{2}$ Wenn W. Sanders (1985) z.B. für das Altniederdeutsche die vier Textgruppen Bibeldichtung, Klein- und Merkversdichtung, kirchliche und weltliche Gebrauchsprosa sowie Glossen unterscheidet und H. Kästner/ E. Schütz/ J. Schwitalla (1984) als Textgliederungsraster für das Frühneuhochdeutsche Alltagswelt, Religion, Wissenschaft und Dichtung nennen, so mögen derartige Klassifizierungsbasen zur Gruppierung der Fülle überlieferter Texte brauchbar sein, sie lassen jedoch nicht deutlich erkennen, welche sprachgeschichtlichen Problemstellungen qua textorientierter Untersuchungen zu bewältigen sind. Folglich sollte es zunächst darum gehen, zentrale Funktionen der historischen Textlinguistik für die Sprachgeschichtsschreibung des Deutschen deutlich zu machen; andernfalls steht Text- bzw. Textsortengeschichte eben in der Gefahr, lediglich verschieden differenzierte Systematiken zur Quellenordnung zu erarbeiten.

Im Zusammenhang dieser Darstellung soll ein zentraler Problembereich germanistischer Sprachgeschichtsschreibung herausgegriffen und in Bezug auf textlinguistische Erkenntnismöglichkeiten dargestellt werden. Es handelt sich dabei um die Frage nach der Formierung der deutschen Kultur- und Hochsprache, die wir Neuhochdeutsch nennen. Bekanntlich beschränken sich die traditionellen Erklärungsversuche zur Konstituierung des Neuhochdeutschen fast gänzlich auf die Nennung grammatischer Faktoren; historische Phonologie, ${ }^{3}$ Morphologie, ${ }^{4}$ Syntax ${ }^{5}$ und Lexikologie ${ }^{6}$ sind daher die

2 Der bereits erwähnte Aufsatz von H. Steger (1984) stellt dabei eine Ausnahme dar, allerdings ist er auch vornehmlich konzeptionell orientiert. Die Analyse von Textsorteninventaren wird im Sammelband mithin im Rahmen einzelner Epochen jeweils gesondert dargestellt. 
klassischen Felder zur Beantwortung der Frage, warum und wie sich deutsche Kulturund Hochsprachigkeit entwickelt hat.

Die entsprechende Erklärungsfolie setzt dabei weithin den Parameter grammatischer Überregionalität als Kriterium hochsprachlicher Existenz an, mithin werden in der Regel Leitvarietäten als Muster von Ausgleichsprozessen beschrieben. Die nachfolgende Argumentation geht jedoch davon aus, daß Hoch- und Kultursprachigkeit nicht nur den zweifellos wichtigen Aspekt überregionaler Inventare voraussetzt, sondern daneben noch ein Bündel anderer Faktoren, von denen hier fünf näher genannt werden sollen: ${ }^{7}$

(a) Literalität:

Allgemeinste und zugleich notwendigste Voraussetzung von Kultur- und Hochsprachigkeit ist die Existenz eines Schriftsystems, das die grammatischen Eigenschaften gesprochener Sprache faßt bzw. spezifische schriftsprachliche Grammatikalität bedingt. Unter diachroner Perspektive erscheint das Verhältnis von oraler und literaler Sprache als beachtenswerter Faktor im Prozeß der Konstituierung des Nhd. Es stellt sich die Frage, ob nicht die Anverwandlung eines normierten Schriftsystems, einer Orthographie und Interpunktion als spezifischer Teilvorgang bei der Herausbildung des Nhd. anzusehen ist.

(b) Multifunktionalität:

Die kommunikative Leistungsfähigkeit in unterschiedlichsten Domänen gesellschaftlicher Organisation ist Kennzeichen jeder Kultur- und Hochsprache. Als sprachgeschichtliche Problemstellung ist in diesem Zusammenhang insbesondere zu untersuchen, welchen Anteil die spätmittelalterliche und frühneuzeitliche Funktionsdifferenzierung des Deutschen bei der Herausbildung des Nhd. hatte.

(c) Intersozialität:

Neben der unter (b) genannten horizontalen, domänenspezifischen Funktionalität einer Sprache ist die kommunikative Eignung in vertikaler Richtung, also bezüglich der sozialen Gruppenstruktur als Voraussetzung von Kultur- und Hochsprachigkeit anzusehen. ${ }^{8}$ Erst eine Sprache, vermöge derer über die Grenzen differenter Wissensniveaus Austausch möglich ist, kann als Medium der allgemeinen nationalen Verständigung entfaltet werden und sichert sprachlich manifestierte nationale Identität.

3 Z.B. die Bescheibung der frnhd. Diphthongierung und Monophthongierung als charakteristische Variante des Frnhd. und Nhd. gegenüber dem Mhd.

4 Als aktuelles Beispiel historischer Morphologie sei M. Habermann (1994) angeführt.

5 Vgl. W. Admoni (1990), A. Betten ([Hg.] 1990).

6 Vgl. D. Wolf (1984), O. Reichmann (1984).

7 Vgl. R. Müller (1991).

8 Zu den Begriffen 'Horizontalität' und 'Vertikalität' in der Lingusitik vgl. S. Wichter (1994). 
(d) Literarizität:

Die literarische Ausbildung des Deutschen ist ausgehend von den fruihen volkssprachigen Texten bis hin zur Existenz eines literarischen Kanons Aspekt der Herausbildung gegenwärtiger Hochsprachigkeit. Für die Sprachgeschichte stellt sich die Frage, welche Quellen des vornhd. Status den aktuellen Sprachstand mitbegründen.

(e) Philologisierung:

In der Entwicklungsgeschichte von Hoch- und Kultursprachen bedingt erst die philologische Reflexion ein Bewußtsein der je eigenen Sprache. Mit der Zunahme an Sprachbewußtsein gehen die deskriptiven und normativen Beschreibungen der Entwicklungsvorgänge jeweiliger Sprachen einher, die sich in Wörterbüchern, Grammatiken, Stillehren etc. niederschlagen. ${ }^{9}$ Sprachgeschichte kann hier nach dem Anteil bewußter Steuerung der sprachlichen Genese durch die Reflexion ihrer Erscheinungsformen fragen.

Jeder dieser Faktoren ist mit Detailaufgaben sprachgeschichtlicher Forschung verbunden, die hier nicht alle in ihrem spezifischen Profil zu erörtern sind. Greift man jedoch z.B. den Aspekt der Multifunktionalität heraus, so ist bereits festzustellen, daß grammatische Erklärungsweisen allein nicht ausreichend zur Beschreibung der Entwicklung dieses Aspektes sind. Denn zu fragen ist hier nach der funktionalen Differenzierung etwa des Deutschen, die als Voraussetzung einer Formierung von Hochsprachigkeit anzusehen ist. Für die germanistische Sprachgeschichtsschreibung geht es in diesem Zusammenhang um die Frage, welche funktionale Differenziertheit das Deutsche infolge der Ausweitung auf kommunikativ zentrale Bereiche entwickelt hat und welches die Ursachen dieser Entwicklung sind. Daß grammatische Ausgleichstheorien dieses Problem nur verkürzt fokussieren können, ergibt sich bereits aus der Beobachtung, daß die Ablösung des Lateinischen als der mittelalterlichen Koiné durch deutsche Volkssprache weit vor der Konstituierung grammatischer Einheitlichkeit des Deutschen beginnt. Die Beschreibung von Entwicklungslinien der Multifunktionalisierung des Deutschen wird damit zum unabdingbaren Bestandteil einer Klärung der Formierung des Nhd. Da Multifunktionalität, also die linguale Funktionstüchtigkeit in unterschiedlichsten Domänen gesellschaftlicher Organisation erst über das Handlungspotential einer Sprache bzw. den kommunikativen Gebrauch sprachlicher Mittel erkennbar ist und Texte i.S. obiger Ausführung als komplexe sprachliche Handlungen anzusehen sind, muß der Text als maßgebliche Größe in der Genese des Nhd. eingeordnet werden. Ebenso wie das phonologische und morphologische Inventar bestimmend für die Herausbildung etwa von überregionalen Varianten ist, ist der Text als entscheidender Parameter der Formierung von lingualer Multifunktionalität anzusehen. Diese Bestimmung der sprachgeschichtlichen Relevanz von Texten trifft sich mit I. 
Rosengrens (1983) Theorie der Textstruktur als einem Ergebnis strategischer Überlegungen des Senders. Hier wird davon ausgegangen, daß die textuelle "Struktur von dem unmittelbaren Ziel des Senders determiniert wird" (Rosengren 1983, 166), womit historische Texte als Realisationsexemplare intendierter sprachlicher Handlungen zu verstehen sind, deren Folge dann eine linguale Funktionsdifferenzierung ist.

Aus dieser Überlegung läßt sich eine der zukünftigen Aufgabenstellungen der historisch-germanistischen Linguistik präzise formulieren: Sie hat zu klären, wann, aufgrund welcher Ursachen und wie multifunktionale Leistungsfähigkeit des Deutschen angelegt wurde und wie die damit verbundenen geschichtlichen Ecksteine im diachronen Prozeß ausgebaut wurden. Eine so verstandene textorientierte Sprachgeschichtsschreibung geht über die bloße Quellensortierung hinaus, sie eröffnet eine pragmatische, weil sprachhandlungsbezogene Perspektive in der z.T. noch immer strukturell dominierten Historiolinguistik. Die Fragen nach den Ursachen und den zeitlichen Bezügen der Entwicklung von lingualer Multifunktionalität sind nun allerdings nicht über die Analyse der textinternen Strukturcharakteristika zu beantworten. Denn konkrete Texte sind in ihrer jeweiligen Realisation immer schon Ergebnisse des u.U. ursächlichen Bestrebens, die deutsche Sprache in zuvor lateinisch besetzten Domänen zu gebrauchen. Die textinterne Organisation ist also nicht Ursache von Multifunktionalisierungstendenzen, sondern Folge derselben. Demgegenüber bezieht sich die Frage nach dem 'wie' der Multifunktionalisierung des Deutschen auf konkrete Texte als denjenigen sprachlichen Einheiten, die eine kommunikative Ausweitung auf weite Bereiche des sozialen Verbandes realisieren. Im folgenden soll daher nicht nach den Ursachen von Multifunktionalisierungstendenzen in der Geschichte des Deutschen gefragt werden, verließe man damit doch unweigerlich den hier interessierenden textlinguistischen Rahmen. Behandelt werden soll vielmehr der Text als Realisationsform von Multifunktionalisierungsprozessen, womit es darum geht, die durch textuelles Handeln unmittelbar determinierten Textkonstituenten ins Auge zu fassen, also jene zentralen universalpragmatischen Strukturen, mittels derer ein Textproduzent seine kommunikative Absicht handelnd realisiert. Zur Bewältigung dieser Aufgabe ist ein leistungsfähiges Textanalysekonzept notwendig, das den Besonderheiten der sprachhistorischen Problemstellung entspricht. Vorschläge zur Konzeption eines solchen sind Gegenstand des weiteren Argumentationsganges.

\section{Konzeption eines pragmatisch orientierten Verfahrens zur Analyse historischer Texte}

Ziel des hier zu erörternden pragmatischen bzw. kommunikationsorientierten Konzepts zur Analyse historischer Texte ist die Freilegung des pragmatischen Potentials einer Sprache zu einem gegebenen Zeitpunkt als Teilaspekt der Erklärung diachroner Vorgänge bei der Konstituierung von Hoch- und Kultursprachigkeit. Beantwortet wer- 
den muß für die deutsche Sprachgeschichte damit zum einen, welche Textkonstituenten die kommunikative Substanz des sprachlichen Handelns ausmachen, also welche pragmatische Organisationsstruktur die an der Multifunktionalisierung des Deutschen beteiligten Texte aufweisen und zum anderen, von welchem Entwicklungsstand des Textypenrepertoires in der Frühgeschichte nhd. Gemeinsprachigkeit auszugehen ist. Die Problemstellung sei zunächst durch ein Beispiel anschaulich gemacht.

Die überlieferten ahd. Quellen gehören vielfach zum Typ der Übersetzungsliteratur, so etwa die in Folge der Admonitio generalis entstandenen Übersetzungen elementarer katechetischer Texte aus dem Lateinischen. Es gelingt den Übersetzern bekanntlich dabei häufig nicht, die lateinischen Vorlagen adäquat zu übertragen. So in den fälschlichen präsentischen Verbformen des 123. Psalms in der alemannischen Psalmenübersetzung (um 820) bzw. der dort ebenso nachzuweisenden Mißachtung des Konjunktivs der Vulgata-Vorlage. ${ }^{10}$ Solche morphologischen Fehlübertragungen führen dann in der frühen ahd. Übersetzungsliteratur u.a. dazu, daß Sprachhandlungstypen und argumentative Muster der lateinischen Vorlage nicht korrekt wiedergegeben werden; die Volkssprache der Übersetzer bzw. die volkssprachige Norm ist kommunikativ undifferenzierter als das Lateinische der Zeit; dem Lateinischen wächst zunächst durch deutsche Volkssprache keine Konkurrenz zu. Dies ändert sich jedoch aufgrund hier nicht zu behandelnder Faktoren im 13. und 14. Jahrhundert, denn gesellschaftlich zentrale Vertextungsbereiche werden volkssprachig besetzt, das Lateinische wird in seiner funktional weiten Fächerung anfänglich zurückgedrängt. Frühe deutsche Fachprosatexte sind Folge dieser Entwicklung, ihre Strukturierung steht infolgedessen im Zusammenhang sprachgeschichtlicher Erkenntnisinteressen.

Die konkreten Problemstellungen im Zusammenhang einer allgemeinen Konzeption zur Beschreibung der pragmatischen Organisationsstruktur von Texten ordnen sich vier konzeptionellen Subbereichen zu:

( $\alpha$ ) Eine Analyse von Texten, die infolge der Entwicklung von Multifunktionalität existieren, hat zu klären, welche illokutive Differenzierung frühe volkssprachige Prosa leistet, denn die sprachliche Realisation der kommunikativen Absicht eines Textproduzenten ist wesentliches Element der pragmatischen Organisation von Texten. Dabei wird davon ausgegangen, daß Texte als über die Einzelillokution hinausgehende Illokutionskomplexe bzw. Illokutionsstrukturen anzusehen sind. ${ }^{11}$ Es besteht also für (a) die Aufgabe, textuelle Dominanzfelder von Illokutionen zu ermitteln. Dabei sind insbesondere die subsidiären Funktionen von Sprechakten auf "macro-speech acts" i.S. T.v.Dijks $(1977,215)$ zu beziehen. Eine solche Illokutionsanalyse, die mit A. Wagner (1994) Sprechakte als historisch variable Größen

10 Vgl. hierzu die Edition des Textes und den Kommentar in W. Haug und B.K. Vollmann [Hg.] (1991, $32 ; 1056)$.

11 Zur Relevanz sprechaktheoretischer Modelle bei der textlinguistischen Analyse vgl. den in diesem Band enthaltenen Aufsatz von Stojan Bračič. 
ansetzt, ermöglicht die Beschreibung einer wesentlichen pragmatischen Textkonstituente.

( $\beta$ ) Als weiterer Beschreibungsbereich ist die propositionale Struktur von Texten zu untersuchen. Die thematische Substanz von Texten, ihr Komplexitätsgrad ist Hinweis auf die kommunikative Leistungsfähigkeit einer Sprache, denn für den Zeitpunkt, wo differenzierte Propositionskomplexe über die Grenzen enger Domänen gesellschaftlicher Organisation textuell gefaßt werden, ist zumindest von einer partiellen Multifunktionalität auszugehen. Die Realisation komplexer und u.U. fachspezifischer thematischer Strukturen mit volkssprachigen Mitteln ist Folge. der Verwendung des Deutschen in zentralen gesellschaftlichen Bereichen seit dem beginnenden Spätmittelalter.

$(\gamma)$ Neben der Illokutions- und Propositionsstruktur ist der argumentative Aufbau von Texten, also die Strukturierung von Behauptungen mit ihren impliziten und expliziten Rechtfertigungen eine weitere Konstituente der pragmatischen Organisation von Texten. Da hier mit K. Brinker $(1983,138)$ davon ausgegangen wird, daß zwischen Sprachhandlung und zugrunde liegendem Muster "prinzipiell kein 1:1Verhältnis besteht", werden Illokutions-/Propositionsstruktur und Argumentstruktur als distinkte Beschreibungsebenen des Textes angesehen. Entsprechend wird davon ausgegangen, daß die argumentative Differenziertheit eines Textes notwendiges Analysefeld für die Beschreibung textueller Strukturierung ist.

Die drei Aspekte $(\alpha-\gamma)$ werden im folgenden als textinterne Konstituenten der pragmatischen Organisationsstruktur angesehen. Der funktionale Entwicklungsstand bedingt aber nicht nur jeweilige textinterne Strukturebenen, sondern steht auch in direktem Zusammenhang mit der Referenz von Texten auf bzw. der Determinierung durch textexterne Faktoren.

( $\delta$ ) Diese sollen hier unter dem Begriff des pragmatischen Kontextes zusammengefaßt werden, worunter eine typologische Größe textexterner Variablen vorzustellen ist.

Die konzeptuellen Subbereiche (a-d) gilt es nun näher zu behandeln, wobei gleich der letzte Punkt konkretisiert werden soll.

\subsection{Pragmatischer Kontext}

Vom pragmatischen Kontext soll gesprochen werden, da Texte in inzwischen weit verbreitetem textlinguistischen Verständnis immer in Bezug zu einem Handlungsumfeld stehen, denn Textualisierung ist linguale Handlung, die notwendigerweise in einem pragmatischen Kontext geschieht. Für die Beschreibung der Beziehung von textueller Handlung und pragmatischem Kontext sind zwei Relationsformen zu unterscheiden. Einerseits determinieren jeweilige situative Umstände die textuelle Handlung, die Organisation von Texten ist mithin als situationsabhängig zu beschreiben. Die situativen Variablen sind dabei auf den Begriff der textuellen Situation bezogen. Andererseits wird durch textuelles Handeln wiederum selbst Bezug auf die pragmatischen Umstände genommen. So wird etwa ein mittelalterlicher Text über die Ständeordnung auf exi- 
stente Privilegskorporationen referieren. Die Zielgröße der Referenz textueller Handlungen auf die sozialen Organisationsformen des pragmatischen Kontextes wird im folgenden als textueller Organisationsbereich bezeichnet. Textuelle Situation und textueller Organisationsbereich bilden zusammen die relationalen Verbindungen zwischen textueller Handlung und pragmatischem Kontext. Die Aspekte selbst stehen in komplementärem Verhältnis zueinander: Der pragmatische Kontext determiniert einerseits die textuelle Handlung durch die textuelle Situation, andererseits referiert die textuelle Handlung auf den pragmatischen Kontext durch Bezug auf einen textuellen Organisationsbereich.

Die Bestimmung des textuellen Organisationsbereichs hat grundsätzlich im Rückgriff auf die im Text explizierten Informationen zu erfolgen. Solche textkonstitutiven Daten können als Variablen in einer Matrix erfaßt werden, wobei es jedoch nicht sinnvoll erscheint, einen deduktiven Katalog zu erarbeiten, der dann auf verschiedene Texttypen zu übertragen ist. Der pragmatische Kontext von Texten und die damit gegebenen Referenzbeziehungen sind zu zahlreich und verschieden, um sie mit einer Generalmatrix erfassen zu können. Institutionelle Texte (Verordnung) haben z.B. wesentlich normiertere Daten der Referenz auf den sozialen Organisationsbereich als private Texte (Liebesbrief), womit auch deren Bestimmung ein anderes Maß an Genauigkeit ermöglicht. Entgegen einem deduzierten Variablenraster wird hier vielmehr von induktiv ermittelten Variablen des sozialen Organisationsbereichs ausgegangen. Diese sind nicht generell zu formulieren, sondern lediglich für sozial komparabel funktionierende Texte als Vergleichsgrößen zu bestimmen. Für die deutschsprachige Rechtsprosa des Spätmittelalters ergeben sich die Variablen etwa aus den Fragen, ob im Text explizite Angaben zur arealen Geltung der Aussagen enthalten sind, wie der sachliche Geltungsumfang der Aussagen, also in der Regel die Zielgruppe des Textes expliziert ist und welche Informationen zur zeitlichen Geltung normierender Rechtsaussagen gegeben sind. Damit ergeben sich räumlicher, sachlicher und zeitlicher Geltungsbereich als mögliche Bezugsgrößen auf den sozialen Organisationsbereich der entsprechenden Rechtstexte. In den jeweiligen Texten sind nun nahezu immer klassenbildende Explikationen zu den Kriterien enthalten, die Texte als Handlungsmuster in einem je spezifischen pragmatischen Umfeld erkennen lassen. Eine differenzierte Bestimmung des sozialen Organisationsbereichs setzt allerdings noch die Formulierung der eigentlichen Variablen voraus, die erst Vergleichbarkeit von Texten eines sozialen Typs (z.B. Rechtstexte) ermöglicht. Nach Anzahl und Verteilung sind diese Variablen heuristische Größen und stehen wie gesagt in enger Bindung an je konkret ausgewertete Texte. Für die Rechtsprosa des Spätmittelalters wäre etwa folgende Darstellung der Variablen in einer Matrix möglich: ${ }^{12}$

12 Unter 1. ist etwa anzugeben, ob es sich um gemeine also auf das gesamte mittelalterliche Reichsgebiet bezogene Rechtssetzung bzw. Auslegung handelt oder um partikulares Recht. Unter (2) ist aufzuführen, ob die Aussagen des Textes eine generelle sachliche Geltung haben, also jede im räumlichen Geltungsbereich lebende Person betreffen, oder ob die sachliche Geltung nur spezifizierte 


\begin{tabular}{|l|l|c|}
\hline 1. räumlicher Geltungsbereich & 1.1 Reich & \pm \\
& 1.2 Region & \pm \\
& 1.3 Stadt & \pm \\
& 1.4 definierter Sonderbereich (Seerecht etc.) & \pm \\
\hline 2. sachlicher Geltungsbereich & 2.1 generell & \pm \\
& 2.2 speziell & \pm \\
\hline 3. zeitliche Geltung & 3.1 bestimmt & \pm \\
& 3.2 unbestimmt & \pm \\
\hline
\end{tabular}

Auf der Grundlage derartiger Matrizes ist eine analytische Zuordnung zu einem textuellen Organisationsbereich möglich. Damit ist eine Relation von textueller Handlung und pragmatischem Kontext erfaßt. Die textuelle Situation als zweite Relationsform resultiert nun wie ausgeführt aus den situativen Variablen, die als textdeterminierende Daten eingeordnet sind. Unter 'Situation' werden hier die expliziten Rahmenbedingungen textuellen Handelns angesehen. Die so verstandene Situation stellt sich dabei allerdings nicht als ungeordnete Vielzahl situativer Komponenten dar, sondern als geordnete Menge situativer Aspekte. Es führte über den hier behandelten Gegenstand weit hinaus, im einzelnen diese Aspekte erläuternd darzustellen. Da es in der momentanen Argumentation lediglich darum geht, Texte hinsichtlich ihres zweifachen Bezuges $\mathrm{zu}$ einem jeweiligen pragmatischen Kontext zu beschreiben, mag es genügen, die anzunehmenden Situationsvariablen unkommentiert in einer wiederum auf spätmittelalterliche Rechtsprosa bezogenen Matrix darzustellen, zumal diese weithin selbsterklärend sind und in der textlinguistischen Diskussion als vertraut gelten dürfen. Erklärend sei nur darauf hingewiesen, daß die einzelnen Daten zur textuellen Situation drei Situationsbereichen zugeordnet sind: Zum Situationsbereich der sozialen Situation gehören alle Determinanten, die die Konnexion des Textes mit dem gesellschaftlichen Handlungsrahmen betreffen; zu erfassen sind damit der Grad gesellschaftlicher und zeitlicher Bindung des Textes, sowie die mögliche Intertextualität. Die soziale Situation ist dabei nicht mit dem textuellen Organisationsbereich zu verwechseln. Während dieser als Referenzgröße des Textes definiert ist, determinieren die Variablen der sozialen Organisation die textuelle Handlung. Gegenüber der sozialen Organisation ergibt sich die formale Situation eines Textes aus dem kommunikativen Status des Textproduzenten und der Wahl der Kommunikationsmittel. Von beiden Situationsbereichen ist die interpersonale Situation unterschieden. Sie ergibt sich aus der für einen Text jeweils bestimmenden Kommunikationskonstellation, also dem Verhältnis von Produzent und

Personengruppen bzw. Einzelpersonen betreffen. Die Variablen unter (3) ergeben sich aus der zeitlichen Bestimmung der Textaussagen, also auf Angaben zur ausdrücklich vorübergehenden Gültigkeit (z.B. Zeitgesetze) und solche zur unbestimmten zeitlichen Geltung; bei vorhandener Zeitbestimmung ist diese in der Regel wesentlicher Bestandteil der Promulgation. 
dem bei Schrifttexten zumeist antizipierten Rezipienten; damit sind die Handlungsakteure selbst als Bestandteile der Situation angesehen. Neben den Situationsbereichen differenziert die Matrix noch zwei prinzipiell unterschiedliche Situationsarten: die variablen, je Text verschiedenen Situationsmerkmale und die konstanten, für alle Texte eines homogenen Korpus zutreffenden Daten. Da für einen historisch abgeschlossenen Zeitraum nur schriftliche Texte untersucht werden können, ergeben sich für die formale Situation als Konstanten der Rechtsprosa des Spätmittelalters das schriftliche Medium, eine monologische Kommunikationsrichtung und eine situationsgebundene Festlegung des Redegegenstandes. Die konstanten interpersonalen Situationen sind ein gebundenes Rollenverhältnis und ein raum-zeitlich differenter Partnerkontakt. Damit sind fünf der zwölf situativen Textdeterminanten konstante Größen, also durch einen für alle Texte eines Korpus der deutschen Rechtsprosa des Spätmittelalters zutreffenden Eintrag gekennzeichnet. Als variable Daten der Matrix sind demzufolge die Aspekte der sozialen Situation zu bestimmen sowie für die interpersonale Situation der soziale Raum, das Verhältnis der Kommunikationspartner, die Sprecherzahl und die hierarchische Relation von Produzent/Rezipient. Als Variable der formalen Situation ist der kommunikative Status des Textproduzenten zu untersuchen.

Die textanalytische Beschreibung der Situation kann demzufolge für spätmittelalterliche Rechtsprosa mittels Differenzierung von drei Situationsbereichen geschehen, denen mindestens 13 Situationsaspekte bei Differenzierung von zwei Situationsarten zugeordnet sind. In der Übersicht ergibt sich die folgende Matrix:

\begin{tabular}{|c|c|c|}
\hline \multirow[t]{2}{*}{ Situationsbereich } & \multicolumn{2}{|l|}{ Situationsaspekt } \\
\hline & variable Situationsart & konstante Situationsart \\
\hline 1. soziale Situation & $\begin{array}{l}1.1 \text { gesellschaftliche Bindung } \\
\text { [keine/schwach/stark] } \\
\text { 1.2 zeitliche Bindung } \\
\text { [vorzeitig/nachzeitig/simultan] } \\
\text { 1.3 Textkonnexion } \\
\text { [belegt/nicht belegt] }\end{array}$ & \\
\hline 2. formale Situation & $\begin{array}{l}\text { 2.1 Status des Textproduzenten } \\
\text { [expliziert/anonym] }\end{array}$ & $\begin{array}{l}\text { 2.2 Themenwahl } \\
\text { [mittelbar] } \\
\text { 2.3 Kommunikationsrichtung } \\
\text { [monologisch] } \\
\text { 2.4 Medium } \\
\text { [schriftlich] }\end{array}$ \\
\hline
\end{tabular}




\begin{tabular}{|l|l|l|}
\hline $\begin{array}{l}\text { 3. interpersonale } \\
\text { Situation }\end{array}$ & $\begin{array}{c}3.1 \text { sozialer Raum } \\
\text { [öffentlich/privat] } \\
\begin{array}{c}3.2 \text { Verhältnis der } \\
\text { Kommunikationspartner } \\
\text { [soziale Nähe/soziale Ferne] }\end{array}\end{array}$ & $\begin{array}{c}3.3 \text { Rollenverhältnis } \\
\text { [gebunden] } \\
\text { 3.4 Art des Interaktionskontaktes } \\
\text { [raum-zeitlich different] }\end{array}$ \\
$\begin{array}{c}3.5 \text { Anzahl der Sprecher } \\
\text { [einer/mehrere] } \\
3.6 \text { hierarchische Relation von } \\
\text { Produzent und Rezipient } \\
\text { [symmetrisch/asymmetrisch] }\end{array}$ & \\
\hline
\end{tabular}

In der Zusammenfassung ist also für den konzeptionellen Subbereich (d) festzuhalten, daß der pragmatische Kontext von Texten in zweifacher Relation zum Textexemplar bzw. zur textuellen Handlung steht: Wir unterscheiden Situationsdetermination zum einen und zum anderen die Referenz auf einen sozialen Organisationsbereich. Die jeweiligen Belegungen der Matrizes sind dabei als Daten zur Bestimmung des pragmatischen Kontexttyps einzelner Texte heranzuziehen. Die binäre Bezugsetzung von textueller Handlung und pragmatischem Kontext widerspricht situationsdeterministischen Textauffassungen, die die Kombination außersprachlicher Textbedingungen als alleinige Bestimmungsgröße für Textsorten, -typen etc. anführen. ${ }^{13}$ Einer solchen Auffassung kann hier schon aufgrund der Integration des sozialen Organisationsbereichs von Texten nicht entsprochen werden. Darüber hinaus spricht gegen einen absoluten Situationsdeterminismus, daß die drei textinternen Strukturcharakteristika $(\alpha-\gamma)$ in Korrelation gedacht werden müssen, die Ebenen 'Illokutions-/Propositions-/Argumentstruktur' sind durch textinterne Binnendetermination gekennzeichnet. Demzufolge wird hier davon ausgegangen, daß eine Texttheorie, die Situationstypen als allein merkmalbildend einordnet, zu kurz greift.

Die weitere Behandlung des kommunikationsorientierten Verfahrens zur Analyse historischer Texte hat nun die konzeptionellen Subbereiche $(\alpha-\gamma)$ näher zu bestimmen.

13 Vgl. etwa das Freiburger Redekonstellations-Konzept. Hier wird der außersprachliche Teil der Situation, der als 'Redekonstellation' bezeichnet wird und "die in einem bestimmten Kommunikationsakt auftretende Kombination außersprachlicher Verhaltenselemente" (Steger et al. 1974, 60) umfaßt als Determinante der 'Textsorten' beschrieben, die ihrerseits typologisches Äquivalent der konkreten 'Textexemplare' sind. Die Texterzeugung ist demzufolge in unmittelbarer und alleiniger Abhängigkeit von den Redekonstellationstypen gesehen.

Als situationsdeterministische Texttheorien sind auch M. Halliday (1978,185), B. Marfurt (1978) und G. Diewald $(1991,271)$ anzuführen. 


\subsection{Illokutionsstruktur $(\alpha)$}

Im Lunder Forschungsprojekt 'Fachsprachliche Kommunikation' (s. Schonebohm 1980) wird davon ausgegangen, daß Grammatik und Pragmatik in -einem modularen Verhältnis zueinander stehen, mithin als autonome sprachliche Komponenten einzuordnen sind. Diese Annahme kommt der hier beabsichtigten Konzipierung eines Modells zur Analyse historischer Texte im Kontext der sprachgeschichtlichen Multifunktionalisierungsvorgänge insofern entgegen, als auch für diese Problemstellung von der prinzipiellen Unterschiedlichkeit grammatischer und pragmatischer Faktoren im Prozeß der Konstituierung des Nhd. ausgegangen wurde. Die modulare Distinktion von Grammatik und Pragmatik ergibt sich im theoretischen Entwurf, doch in Texten agieren Grammatik- und Pragmatikmodul miteinander, so daß bei der Analyse des Funktionspotentials zunächst die Konstituenten des Pragmatikmoduls bestimmt werden müssen. M. Brandt und I. Rosengren (1992) gehen davon aus, daß als zentrales Submodul des Pragmatikmoduls das Illokutionssystem anzusetzen ist. Im folgenden wird dieser Bestimmung gefolgt, da Illokutionen - i.S. kommunikativer Absichten eines Produzenten als wesentliche Indikatoren des funktionalen Handlungspotentials einer Sprache zu verstehen sind. Dabei wird davon ausgegangen, daß die Ausformung von Sprechaktklassen und ihre interne Differenziertheit im Ausschnitt erkennen lassen, mit welchen kommunikativen Mitteln eine Sprache operiert.

Wenn nun im Zusammenhang der hier beabsichtigten textanalytischen Konzeption von Illokutionen gesprochen wird, so sind damit allerdings nicht singuläre Komponenten von Sprechakten gemeint, sondern vielmehr textuell verknüpfte Sprechaktsequenzen. Denn erst die sequentielle Organisation von Illokutionen ist Hinweis auf die kommunikative Realisation allgemeiner Intentionen sprachlicher Handlung; der Zweck einer Einzelillokution wird erst relational zum illokutionären Kontext erkennbar. Die sprachliche Handlung eines Sprechers wird daher, sofern kein initialer Sprechakt vorliegt, im relationalen Bezug zu vorausgehenden Sprechakten organisiert sein; dies ist zunächst eine triviale Erkenntnis. In Bezug auf die hier leitende Problemstellung bedeutet dies allerdings, daß nicht die summarische Erfassung von singulären Illokutionen Erkenntnisse zur textuellen Strukturierung ermöglicht, sondern erst die Analyse der relationalen Verknüpfung von Illokutionen, jedenfalls wenn wir in diesem Zusammenhang davon ausgehen, daß linguale Multifunktionalität die sprachliche Existenz differenzierter und auch domänenspezifischer Illokutionsarchitekturen bestimmt. Also in nuce: Illokutionen stellen in der Regel keine Unikate dar, sondern sind als im Text hierarchisch aufeinander bezogene Einheiten zu untersuchen, womit die historische auf den Zusammenhang von Text und Multifunktionalität fokussierte Textlinguistik die Illokutionsarchitektur historischer Texte zu untersuchen hat. Diese ergibt sich aus den strukturbildenden Relationen der singulären Illokutionen, so daß auch von Illokutionsstrukturen gesprochen werden kann (vgl. Brandt/Rosengren 1992, 13). Sie sind im Text durch ein hohes Maß interner subordinativer Abhängigkeit gekennzeichnet, so daß die einzelnen Illokutionen in der Regel keine koordinativen Verbände bilden, sondern viel- 
mehr Hierarchien. Die kommunikative Absicht eines Sprechers wird also zumeist via Reihung von Einzelillokutionen vorgenommen, die miteinander solche übergeordneten Illokutionen konstituieren, denen der Illokutionszweck einer textuellen Handlung entspricht. Mithin realisiert sich der generelle Handlungszweck der Textformulierung über die Bezugsetzung einzelner, spezieller Illokutionen zu diesem übergeordneten Ziel. Bekanntlich sind in der Textlinguistik derartige textdominante Illokutionen, die den Handlungszweck des Gesamttextes realisieren, den "macro-speech acts" (van Dijk 1977, 215) oder dem "global speech-act" (ebd., 238) bzw. dem "dominierenden Handlungstyp" (Motsch/Viehweger 1981, 140) zuzurechnen. Sowohl T. van Dijk als auch W. Motsch/ D. Viehweger gehen dabei in ihren diesbezüglichen Entwürfen von subsidiären Konstituenten der übergeordneten Sprechakte aus:

"We may have sequences of speech-acts, but some of such sequences may be interpreted as one speech act, consisting of several component or auxiliary acts. (van Dijk 1977, 215)

"Ein Text wäre somit eine Folge von 1-n pragmatisch verknüpften Sätzen oder Satzkomplexen, von denen ein Satz oder Satzkomplex die kommunikative Funktion des gesamten Textes bestimmt und die übrigen subsidiäre Funktion erfüllen." (W. Motsch/D. Viehweger 1981, 140)

W. Motsch/ D. Viehwegers hier formulierte Auffassung der unikalen Realisation des dominanten Sprechaktes über einen Satz bzw. Satzkomplex ist modifiziert in W. Koch/I. Rosengren/M. Schonebohm (1981), wo von mehreren diktiven Handlungen ${ }^{14}$ ausgegangen wird. Den Entwürfen gemeinsam ist die Modellierung von Sprechaktbzw. Illokutionshierarchien und ihren subsidiären Konstituenten.

Die entsprechenden textuellen Strukturen gilt es also zu berücksichtigen, wenn Texte in den Zusammenhang einer funktionsorientierten Erklärung sprachgeschichtlicher Vorgänge gestellt werden. Das Ziel sollte eine korpusbezogene Deskription i1lokutiver Strukturen im beschriebenen Sinn sein, die Antworten zu den historischen Fragen nach dem Zusammenhang von sprachlicher Funktionalität und der jeweiligen Strukturierung von Illokutionen durch koordinative oder subordinative Verknüpfung ${ }^{15}$ ermöglicht. Im konkreten Vorgehen muß es also darum gehen, die Einzelillokutionen ausgehend von illokutionsmarkierenden Einheiten wie z.B. den Interrogativpronomina, Modalverben und insbesondere den performativen und referierenden Ausdrücken auf dominante Illokutionen zu beziehen. So ist die im Mainzer Landfriedensgesetz von $1235^{16}$ dreizehnmal wiederholte Formel "Wir Sezzen und gebiten" als explizit performativer Ausdruck zugleich Äußerungsform des direktiven Handlungstyps GEBIETEN und soll die untereinander zu einer funktionalen Einheit verbundenen Illokutionen umfassen." 
des deklarativen Handlungstyps IN KRAFT SETZEN. Die Textanalyse ergibt nun, daß eine Vielzahl untergeordneter, da argumentativ subordninierter Sprechakte die direktive Handlung stützen, so daß GEBIETEN als dominante Illokution anzusetzen ist, deren Zweck (illocutionary point) dahingeht, den Textadressaten auf eine zukünftige Handlung festzulegen. Der mit der Promulgation einhergehende deklarative Sprechakt - der zwar die proklamatorische Funktion des Textes indiziert (vgl. E.U. Große 1976, 60) ist gegenüber dieser Absicht indirekt, denn die Direktion wird hier einfach qua Deklaration vollzogen. Dies ändert nichts am zentralen Illokutionszweck des Textes, der in der Aufforderung an den Empfänger liegt, einer Anweisung Folge zu leisten. Die Dominanz dieses Ziels läßt sich übrigens u.a. auch an der hochfrequenten Verwendung des Modalverbs 'sollen' ablesen: "der richter Sal en in die achte twn der den vride gebrochin hat" (16,26f) "tut des der richter nicht das fal der keifer richten alfe recht ift" $(16,43 f)$. Der gesellschaftlichen Funktion des Landfriedensgesetzes, soziale Gegenbenheiten positiv verändern zu wollen, entspricht die beschriebene Relation von Direktiva und Deklarationen vollständig.

Wie an diesem Ausschnitt empirischer Analyse bereits erkennbar wird, ist die Beschreibung von illokutiven Dominanzfeldern und der Okkurenz spezifischer Stützungsrelationen unmittelbar auf die Frage nach der funktionalen Differenziertheit einer Sprache zu beziehen. So bedingt eben die volkssprachige Rechtsbildung und damit die funktionale Relevanz des Deutschen für die Formulierung juridischer Normen z.B. pragmatische Potentiale, ohne die eine sachgemäße, überzeugende Aussage nicht möglich wäre. Die Verkündung des Mainzer Reichslandfrieden in deutscher Sprache auf dem Reichstag im August 1235 und das gleichzeitige Existieren einer lateinischen Fassung, "die als amtlicher Text ... in staufischer Zeit angesehen und verwendet wurde" (Buschmann 1988, 110) ${ }^{17}$ verdeutlicht die funktionale Relevanz des Deutschen im konkurrierenden Verhältnis zum Lateinischen.

Bezüglich der Illokutionstruktur historischer Texte des Deutschen sollte es also zunächst darum gehen, Illokutionskomplexe bzw. -zentren als Nuclei des textuellen Handlungsziels auf eine bzw. einige dominante Illokutionen zu beziehen. Die Textorganisation ist dann darauf zu untersuchen, welche Illokutionsrelationen diese kommunikative Absicht des Textes stützen. Auf diese Weise kann ein wesentliches Submodul der pragmatischen Bedingungen sprachlicher Entwicklung erfaßt werden, wobei in diesem Zusammenhang auch B. Schlieben-Langes $(1976,114)$ These neu zu überdenken

17 Zu den Problemen der deutschen und lateinischen Fassung des Mainzer Reichslandfrieden vgl. A. Buschmann (1988).

R. Schmidt-Wiegand (1992) hat dargelegt, daß vieles dafür spricht, daß die Verdeutschung des Textes "zum Zwecke der allgemeinen Verkündung" (ebd., 355) aufgeschrieben wurde. Daß die vorliegende deutsche Fassung diesem Ursprungstext nicht entspricht, braucht hier nur am Rande vermerkt zu werden, weil es für die Verwendung des Textes im Argumentationszusammenhang dieses Aufsatzes nebengeordnet ist. 
ist, wonach es "keine universellen sprachlichen Handlungen" gibt, "sondern nur je historisch bestimmte, unterschiedene, konventionalisierte sprachliche Handlungen".

\subsection{Propositionsstruktur ( $\beta$ )}

Zur illokutionären Rolle eines Textes gehört im funktionalen Verständnis von Texten immer auch eine propositionale Struktur. Diese wird im vorgestellten Konzept mithin auch als zweite Konstituente der pragmatischen Struktur von Texten behandelt. Wie bereits für den strukturbildenden, relationalen Bezug von singulären und textdominanten Illokutionen ausgeführt, ist auch bezüglich der Propositionen eines Textes von hierarchischen Beziehungen auszugehen, die sich aus dem textspezifischen Verhältnis von Einzelpropositionen über Teilinhalte bis zum Textthema ergeben. Entsprechend geht es wie bei der Analyse von Illokutionstrukturen darum, strukturelle Hierarchien für den thematischen Gehalt von Texten aufzudecken, determiniert dieser doch den inhaltlichen Komplexitätsgrad eines Textes bzw. indiziert die zeitgebundenen Strategien zum Konnex von Einzelpropositionen. Wesentliches Ziel ist es dabei, die im textuellen Prädikat-Argument-Verband realisierten Propositionen auf die inhaltliche Makroebene des Textthemas zu beziehen. Dazu werden Propositionen als kleinste Einheiten der thematischen Textebene aufgefaßt (vg1. Koch/Rosengren/Schonebohm 1981, 170) deren Konstituenten Prädikate und valenzgebundene Argumente sind. Solche singulären Propositionen bilden nun i.d.R. mikrothematische Komplexe bzw. Teilinhalte von Texten, die wiederum zusammengenommen den Textinhalt i.S. des auf einen oder mehrere Gegenstände bezogenen Gedankengangs eines Textes bilden. Das Textthema ist dann als Kern des Textinhaltes zu formulieren. ${ }^{18}$ Wie an dieser Stufung bereits zu erkennen ist, ergibt sich der thematische Gehalt eines Textes nicht immer unmittelbar aus den Relationen einzelner Propositionen. So weisen etwa komplexe Texte, mit denen bei der historischen Analyse gerechnet werden muß, in der Regel nicht nur ein Thema auf, ihr Informationsgehalt ist vielmehr oft heterogen, also durch Komposition von Subthemen bzw. Teilinhalte organisiert. In solchen Fällen ergibt sich der thematische Gehalt eines Textes zwar auch ausgehend von singulären Propositionen, doch entscheidend ist der analytische Schritt über die textuellen Subthemen. Die propositionalen Strukturen von Texten realisieren sich mithin oft als hierarchischer Verbund von Ober- und Nebenthemen. Nochmals am Beispiel des Mainzer Landfriedens von 1235 sei das Verfahren in aller Kürze verdeutlicht: Das Textthema wird hier durch 13 Subthemen realisiert, die ihrerseits teilweise bis zu sieben mikrothematische Komplexe enthalten, die über einzelne Propositionen realisiert sind. Geht man z.B. von den Ausführungen "Von des keisers hoferichtere" (17, 7-34) aus, die thematisch besonders dicht organisiert sind, so ist erkennbar, wie die singulären Propositionen zunächst subthematische Einheiten erster Ordnung bilden: 


\begin{tabular}{|ll|l|}
\hline \multicolumn{2}{|l|}{ singuläre Propositionen } & subthematische Einheit 1. Ordnung \\
\hline 1 & Hof hat Hofrichter & \\
\cline { 1 - 2 } & Hofrichter ist freier Mann & \\
\cline { 1 - 2 } & Hofrichter ist im Amt & \\
\cline { 1 - 2 } & Hofrichter sitzt zu Gericht & \\
\cline { 1 - 2 } & Hofrichter behandelt Klagen & \\
\cline { 1 - 2 } & Hofrichter richtet allein & \\
\hline
\end{tabular}

Diese erste Stufe der Teilinhalte konstituiert wiederum subthematische Einheiten höherer Ordnung, so daß sich eine hierarchische Inhaltsstruktur ergibt, die als Baumgraph darzustellen ist; hier wird nur ein Ausschnitt der Inhaltshierarchie des Mainzer Landfriedens wiedergegeben:

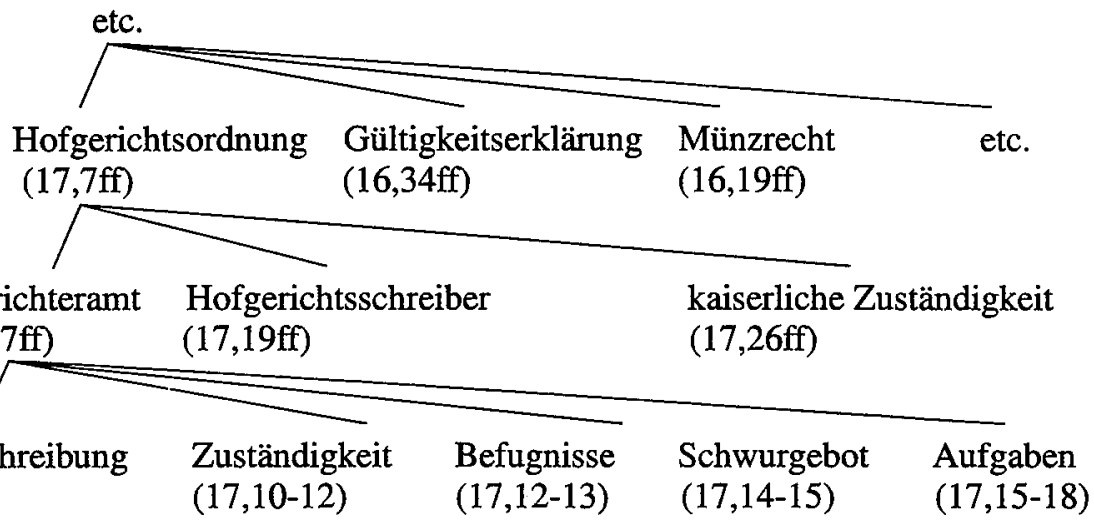

An der Spitze des Baumgraphen hat das Thema als inhaltlicher Kern der gesamten propositionsbasierten Struktur zu stehen. Dessen Formulierung ist allerdings selten per Transformation von Textaussagen zu leisten, wenn auch evtl. existente Teil- und Gesamtüberschriften die Funktion thematischer Zusammenfassungen haben können. Der hierarchischen Struktur thematischer Substanzen entsprechend, wird in den meisten Texten das Thema nicht explizit genannt. Es ergibt sich in solchen Fällen erst als diskrete Bezugsgröße der propositionalen bzw. teilinhaltlichen Stufung in Folge einer Inhaltsanalyse und ist insofern Produkt eines wissenschaftlichen Prozesses. Da so ermittelte Inhaltskerne je Text neu zu formulieren sind und die thematischen Strukturen weit mehr vom Gegenstand der Textaussagen als von prädeterminierenden Strukturcharakteristika abhängen, wird hier kein formalisiertes Verfahren zur Themenformulierung vorgeschlagen. Hier kann Brinker $(1980,139)$ gefolgt werden, der für die Bestimmung des Themas als Inhaltskern eine "'mechanische' Prozedur' ..., die nach endlich vielen Schritten automatisch zur Themenformulierung führt", ausschließt. Viel- 
mehr sollte die Bestimmung des Themas in Abhängigkeit von den singulären Inhaltsstrukturen jeweiliger Einzeltexte erfolgen. Als Bedingung an dessen Formulierung muß lediglich das Prinzip der hierarchischen Dominanz gestellt werden. Ein Thema muß danach immer die subordinierten Inhaltskomponenten dominieren. Ist dies nicht gegeben, weil Teilinhalte nicht als Teilmengen eines Themas zu verstehen sind, muß von einem polythematischen Text ausgegangen werden; mehrere thematische Richtpunkte dominieren dann verschiedene Inhaltshierarchien.

Für die Prüfung inhaltlicher Hierarchien, gleich ob mono- oder polythematisch organisiert, muß nun bedacht werden, daß die Analyse ihre Grenzen in der Existenz von thematischen Strukturen hat. Denn Texte weisen durchaus nicht zwangsläufig linearkonstante Themenstrukturen auf, beispielweise können Redundanzen oder kommunikationssichernde Formeln etc. für die thematische Substanz irrelevant sein. A. Lötscher (1987) zeigt eine Reihe von Faktoren auf, die Texte als inkohärente thematische Gebilde erscheinen lassen und kommt zu dem Schluß:

"Alle diese Überlegungen zeigen, daß es kein sinnvolles Ziel sein kann, jeden Text vollständig mit allen seinen Teilen als vollkommen kohärente thematische Struktur darstellen zu wollen. In einem Text kann nur eine thematische Struktur gefunden werden, soweit er überhaupt eine aufweist." (Lötscher 1987, 232)

Es ergibt sich auch aus diesen Aussagen, daß die Verfahren zur Analyse von propositonalen bzw. thematischen Strukturen in jedem Fall offen für die je spezifischen Erscheinungsformen historischer Texte sein sollten. Auf diesem Weg ist die Analyse von gestuften Inhaltsorganisationen empirisch adäquat zu leisten.

Die Auseinandersetzung mit Multifunktionalisierungstendenzen in der Geschichte der deutschen Sprache sollte derartige thematische Hierarchieverbünde als historische Kommunikationsverfahren systematisch beschreiben, sind diese doch Beobachtungsfeld für den Entwicklungsstand an thematischer Multifunktionalität. Ebenso wie textspezifische Illokutionsstrukturen als Indikatoren der sprachlichen Verarbeitung von Handlungszielen anzusehen sind, sagt die sprachliche Bewältigung propositionaler Hierarchien etwas über den sachlichen Verwendungshorizont einer Sprache aus. Die thematische Kohäsionen von Teil- bzw. Gesamtinhalten, die thematische Dichte von Texten usw. sind Indikatoren für das kommunikative Funktionieren einer Sprache in einem gegebenen historischen Zustand einer Sprache, ihre Untersuchung ist mithin Bedingung der Möglichkeit von Aussagen zum historischen Prozeß der Multifunktionalisierung des Deutschen.

\subsection{Argumentstruktur}

Als dritte Konstituente der pragmatischen Organisation von Texten wird hier die Argumentstruktur angesehen. Dabei wird davon ausgegangen, daß subsidiäre Illokutionen und subordinierte Elemente des Textthemas in ihrem Bezug zu jeweiligen uibergeordneten Hierarchiestellen auch im Funktionszusammenhang von Begründungsstrategien zu sehen sind. Darauf hat I. Rosengren $(1987,181)$ hingewiesen, die 
Begründungen als sprachliche Operationen bezeichnet, "die auf den Einheiten des Textes, den IR [illokutiven Rollen] und PS [propositionalen Strukturen] operieren."

Ganz allgemein sind Argumentationen immer Verfahren zur überzeugenden Vermittlung eines kommunikativen Anspruches, der durch illokutive Rollen und propositionale Gehalte bzw. thematische Hierarchien realisiert ist. Argumente sollen also immer den Anspruch eines Textproduzenten auf Gültigkeit der von ihm formulierten Aussagen sichern und den Textrezipienten von der Richtigkeit des Gesagten überzeugen. Bedingung der Möglichkeit dazu ist eine sachgemäße Versprachlichung von Argumenten. Steht damit die Notwendigkeit argumentativer Verfahren in Abhängigkeit vom Anspruch, eigene Aussagen zu stützen, so finden sich sprachlich gefaßte argumentative Strukturen natürlich nicht in allen Texten. Ein religiöses Bekenntnis weist z.B. in der Regel keine Argumente für die Gültigkeit der Aussagen auf, wohingegen etwa ein wissenschaftlicher Text i.d.R. substantiell argumentativ ist. Allerdings ist die Okkurenz argumentativer Strukturen nicht an spezifische Domänen gebunden. Das zeigt unter historischer Perspektive gerade die Existenz stringent argumentierender Texte im an und für sich glaubenszentrierten Bereich von Theologie und Religion. So ist es das Ziel vieler scholastischer Texte, den Anspruch auf Wahrheit religiöser Aussagen qua Argument zu sichern. Thomas von Aquin führt etwa in der $»$ Summa contra Gentilis ${ }^{19}$ aus, daß die argumentative Stützung der Existenzbehauptung Gottes, also der Gottesbeweis möglich ist, auch wenn es Theologen gäbe, die allein den Weg des Glaubens und der Offenbarung als Existenzüberzeugung anerkennen:

Dicunt enim quod Deum esse non potest per rationem inveniri, sed per solam viam fidei et revelationis est acceptum. 20

Ostenso igitur quod non est vanum niti ad demonstrandum Deum esse, procedamus ad ponendum rationes quibus tam philosophi quam doctores Catholici Deum esse probaverunt. ${ }^{21}$

Es folgt aus dem Beispiel, daß prinzipiell jede Aussage qua Argumentation gestützt werden kann, argumentative Strukturen mithin potentielle Konstituenten jedes Textes sind. Thomas von Aquins Gottesbeweise werden nun in lateinischer Sprache textuell realisiert. Die Beweisschritte und argumentativen Stützungsrelationen der entsprechenden Texte zeigen das Lateinische dabei als geeignete Sprache für die Handlungsziele des Autors. Betrachtet man vor diesem Hintergrund nochmals die sukzessive

19 Thomas von Aquin: Die Gottesbeweise in der "Summe gegen die Heiden" und der "Summe der Theologie". Text mit Übersetzung, Einleitung und Kommentar herausgegeben von Horst Seidel. Lateinisch-Deutsch. 2., verb. und erw. Aufl. Hamburg 1986.

20 Sie behaupten nämlich, es könne nicht durch die Vernunft gefunden werden, daß Gott ist, sondern dies sei allein auf dem Wege des Glaubens und der Offenbarung angenommen. (ebd., 10f)

21 Nachdem wir also gezeigt haben, daß es nicht vergeblich ist, sich um den Beweis zu bemühen, daß Gott ist, wollen wir zu den Gründen übergehen, mit denen sowohl die Philosophen als auch die katholischen (Lehrer) bewiesen haben, daB Gott ist. (ebd., 14f) 
Ablösung des Lateinischen in gesellschaftlich zentralen Bereichen durch deutsche Volkssprachigkeit seit dem beginnenden 13. Jahrhundert, so ist davon auszugehen, daß das Deutsche infolge der Multifunktionalisierung zunehmend komplexe fachspezifische Argumentationsstrukturen textuell zu fassen hat. Denn die Multifunktionalisierungstendenzen des Deutschen seit dem 13. und 14 Jahrhundert bedingen die volkssprachige Realisierung argumentativer Stützungen von Aussagen. Gerade die Ausweitung des Deutschen im gesellschaftlich zentralen Rechtsbereich - der im übrigen auch heute noch substantiell durch argumentativ dichte Texte repräsentiert ist - deutet darauf hin.

Die Textanalyse zeigt, daß manche frühen volkssprachigen Fachprosatexte tatsächlich argumentativ differenzierte Strukturen aufweisen. So kann davon ausgegangen werden, daß die sprachliche Realisierung argumentativer Strukturen eine der Folgen von Entwicklungstendenzen zur Multifunktionalität ist: Eine Sprache, mittels derer nicht komplex argumentiert wird, ist kommunikativ nur sehr eingeschränkt verwendet. Mithin ist eine Analyse der sprachlichen Verarbeitung von Argumenten unabdingbarer Bestandteil der auf Multifunktionalisierungstendenzen gerichteten historischen Textlinguistik.

Bevor auf die Elemente und Relationen solcher Strukturen einzugehen ist, soll kurz auf den Zusammenhang zwischen Argumenten und Sprechaktklassen eingegangen werden. Im engen Verständnis von 'Argumentation' bezieht sich die Stützung von Aussagen auf Repräsentativa, insbesondere auf Assertionen. Deren Akzeptanz, d.h. das Wissen um Gültigkeit setzt immer eine Argumentation voraus. Das heißt jedoch nicht, daß Argumentationen nur zur Stützung von Behauptungen fungieren können. In einem weiteren Verständnis von 'Argumentation' weisen insbesondere auch direktive Sprechakte argumentative Stützungsstrukturen auf. So muß auch eine Anordnung generell begründbar sein, sie hat nur auf der Basis von Begründungen überzeugende Gültigkeit und erreicht oft nur ihr Ziel, wenn entsprechende Argumente ihren Anspruch rechtfertigen:
A: "Spring aus dem Fenster!"
B: "Warum?"
A: "Es ist die einzige Möglichkeit dein Leben zu retten, das Haus brennt."

Bei fehlender Begründbarkeit sind Direktiva immer anfechtbar. Damit sind argumentative Strukturen also hier als Konstituenten von Texten mit unterschiedlichen dominanten Illokutionen gekennzeichnet.

Was nun die Beschreibung der Elemente und Relationen von textuell präsenten Argumenten angeht, zeigt sich das aristotelische Syllogismusmodell [Prämisse 1/ Prämisse $2 \Rightarrow$ Konklusion] als ungeeignet für die Deskription textueller Argumente, ist deren Struktur doch oft komplexer. Benötigt wird ein Argumentationsmodell, das die hierarchische Stufung subsidiärer Elemente bei der Begründung von Aussagen berücksichtigt. Ein solches - das haben u.a. K. Brinker (1983) und insbesondere L. Huth 
(1975) aufgezeigt - liegt mit St. Toulmins (1975) sechsstufigem Argumentationsmodell vor. Geht St. Toulmin auch von der argumentativen Stützung von Repräsentativa aus, so folgt aus dem bereits Gesagten, daß seine hier als bekannt vorausgesetzten Argumentationspositonen mutatis mutandis auch auf andere Sprechaktklassen übertragbar sind. Was St. Toulmin daher über die Gültigkeit von Behauptungen sagt, ist z.B. auch auf Direktiva zu beziehen:

"Wie bei einem Rechtsspruch hängt die Tauglichkeit des Geltungsanspruchs einer Behauptung ab von der Tauglichkeit der Argumentation, die man zu seiner Stützung vorbringen könnte - ..." (Toulmin 1975, 17)

Für die Auseinandersetzung mit den Folgen von Multifunktionalisierungstendenzen ist dieser Aspekt deshalb so wichtig, weil eine Auswirkung der Multifunktionalität von Sprache die Existenz von sprachlichen Mitteln für die angemessene Architektur von Begründungszusammenhängen ist. $\mathrm{Da} \beta$ Toulmins Argumentationslayout dabei geeignet für die empirisch orientierte sprachgeschichtliche Arbeit ist, sei an einem letzten Beispiel verdeutlicht.

Der Mainzer Landfrieden ${ }^{22}$ spricht eine generelle Verpflichtung zur Zeugenschaft in Angelegenheit des gebotenen Generationenfriedens aus $(15,11 \mathrm{ff})$, die richterlich erzwungen werden kann (14,38f). Diese gesetzliche Bestimmung ist entsprechend der bereits erwähnten Sicherung des gebotenen Vollzugs von Direktiva durch Daten, Schlußregeln, Operatoren etc. gestützt und entspricht exakt St. Toulmins Argumentationspositionen. Der argumentative Anspruch ist dabei die Anordnung der generellen Zeugenpflicht, die der aristotelischen Konklusion [K] entspricht. Als argumentative Daten [D] führt der Mainzer Landfrieden ex negativo an, daß fehlende Zeugen eine Verurteilung verbieten (14,20ff). Dieses Argument ist im Text als Ausnahmebedingung einer weiteren, im folgenden noch beschriebenen Argumentation realisiert. Das argumentative Datum stützt die Konklusion aufgrund einer Schlußregel [SR], wonach Straftaten aufgrund von Zeugenaussagen zu bestrafen sind (14,20ff). Die argumentative Stützung [S] dieser Schlußregel ergibt sich aus dem Gesetzescharakter des Mainzer Landfriedens $(14,11 \mathrm{f})$. Dem entspricht der modale Operator »notwendigerweise «, der durch die Ausnahmebedingung des Schwurs eigenen Nichtwissens $(14,39 f)$ relativiert ist. Die Argumentation lautet also: Da bei Fehlen von Zeugen die Verurteilung einer Straftat verboten ist [D], denn Straftaten gelten entsprechend der gesetzlichen Regelung [S] nur aufgrund von Zeugenaussagen als bewiesen [SR], besteht notwendigerweise [O] eine generelle Verpflichtung zur Zeugenschaft [K], es sei denn, ein Schwur des Nichtwissens entbindet davon $[\mathrm{AB}]$. Die nachfolgende Darstellung zeigt das entsprechende Argumentationslayout nach Toulmin, wobei die graphische Darstellung zu erkennen gibt, daß das Datum dieser Argumentation als Ausnahmebedingung einer weiteren Ar- 
gumentation realisiert ist. Diese nennt als Datum [D] den Fall, daß ein Sohn seinen Vater getötet hat $(14,28 \mathrm{ff})$ woraus entsprechend der Schlußregel [SR] - jeder Sohn der seinem Vater nach dem Leben trachtet ist gesetzlos $(14,32 \mathrm{f})$ - aufgrund der Stützung [S] des geltenden Rechts $(14,11)$ durch den Operator [O] »notwendigerweise « vermittelt die Konklusion $[\mathrm{K}]$ folgt, daß der Sohn gesetzlos ist $(14,32)$, es sei denn, es greift die Ausnahmebedingung [AB] fehlender Zeugen $(14,20 \mathrm{ff})$ zum Beweis der Straftat. An dieser Argumentation ist deutlich zu erkennen, daß bereits in frühen volksprachigen Fachprosatexten die argumentative Struktur komplexer als ein Syllogismus sein kann. Der Text argumentiert eben nicht nach dem Muster "Jeder Sohn der seinen Vater tötet ist gesetzlos/ Sohn hat seinen Vater getötet $\Rightarrow$ Sohn ist gesetzlos". Vielmehr wird der Modus Ponens

Sohn hat Vater getötet (p), wenn Sohn Vater getötet hat dann ist er gesetzlos $(p \rightarrow q)$, also ist Sohn gesetzlos $(\vdash \mathrm{q})$.

durch die Stützung des geltenden Rechts sowie durch Operator und Ausnahmebedingung ergänzt. Überdies zeigt die Darstellung die gestufte Konnexion verschiedener Argumentationen. Allerdings entspricht der argumentative Aufbau nicht der Linearität des Textes, der durch ein hohes Maß an Verweisen gekennzeichnet ist. Anaphorische Formulierungen wie "... wirt he des vor fime richtere vorzuget alse hi vor gefcribin ist ..." $(14,30 \mathrm{ff})$ oder "... an allen difen fachen di hi vor gef chribin fint ..." $(15,11)$ bzw. kataphorische Hinweise wie z.B. "Dis recht ist do von das fich nimant Selbe reche" $(15,28)$ sind dabei ein Mittel der Textkomposition. Die Darstellungen des Argumentslayouts von Texten sollte demzufolge nicht als Textpartitur gelesen werden, denn die rekonstruierten Argumentationsstrukturen entsprechen nicht zwangsläufig der linearen Textgestalt. 
Wer seinem Vater

nach dem Leben trachtet

ist gesetzlos (SR)

Zeugen fehlen $(\mathrm{AB})$

aufgrund des gesetzten

Rechts (S)

fehlende Zeugen

verbieten

Verurteilung (D) notwendigerweise $(0)$ - es besteht eine generelle

Verpflichtung zur

Zeugenschaft (K)

Straftaten gelten nur durch Zeugenaussage als erwiesen (SR)

aufgrund des gesetzten

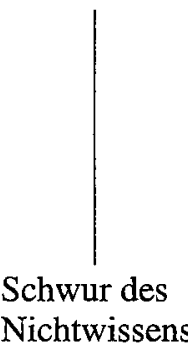

Rechts (S)

Derartige komplexe juristische Argumentationsstrukturen in frühen deutschsprachigen Fachprosatexten sind also ebenso eine Folge der funktionalen Ausweitung des Deutschen auf den gesellschaftlich fraglos zentralen Rechtsbereich, wie die textuelle Realisation von komplexen, meist direktiven Illokutionen und der propositionsbasierte, thematische Bezug von Texten auf rechtliche Gegenstände bzw. Sachverhalte. Es sollte deutlich geworden sein, wie die damit bestimmten universalpragmatischen Textkonstituenten als historisch variable Größen in der Geschichte des Deutschen in Abhängigkeit von Mukltifunktionalisierungstendenzen ausgebildet sind. Illokutionen, Propositionen und Argumente sind natürlich der Möglichkeit nach überzeitliche Strukturcharakteristika aller Texte, doch stehen sie eben im Bedingungsfeld sprachgeschichtlicher Prozesse, womit der Text über seine universalpragmatischen Konstituenten im Kontext jeweiliger textexterner Bedingungen zu sehen ist; dies ist im vorgestellten Analysemodell durch die Typisierung des pragmatischen Kontextes berücksichtigt.

Soweit der Abriß zum Konzept eines kommunikationsorientierten Verfahrens zur Analyse historischer Texte.

Von den anfangs dargestellten drei Desideraten textorientierter Sprachgeschichtsschreibung bleibt damit nur noch etwas zur Frage nach dem Zusammenhang der Ausbildung eines Textbereiches und der Konstituierung der nhd. Kultursprache zu sagen; dies kann abschließend nur in aller Kürze geschehen. 
Für die Herausbildung von Multifunktionalität kommt dem Rechtsbereich besondere Bedeutung $\mathrm{zu}$, das haben die vorangehenden Beispiele bereits andeuten können. Nicht nur, daß wir hier die ersten volkssprachigen Fachprosatexte finden, darüber hinaus kann davon ausgegangen werden, daß die präskriptive Steuerung von Rechtsnormen auch sprachlich normierend wirkt, denn die dem Rechtsbereich eigene Intention, verbindliche Reaktionen beim Kommunikationspartner hervorrufen zu wollen, setzt explizite sprachliche Handlungsmuster voraus. Neben dieser qualitativ herausragenden Stellung der Rechtstexte spricht ein quantitatives Argument für eine textlinguistisch fundierte Analyse von Rechtsquellen: Rechtstexte bilden den größten spätmittelalterlichen Textblock, das funktionale Inventar der Rechtsquellen ist damit als zentrale Größe im Prozeß der Funktionsdifferenzierung des Deutschen anzusetzen. Es spricht somit einiges dafür, den eingangs beschriebenen Praxis-Theorie-Bruch der historischen Textlinguistik u.a. dadurch zu überbrücken, daß für den Rechtsbereich eine exemplarische Analyse der durch die Multifunktionalisierung des Deutschen bedingten Texte entsprechend der vorgestellten Konzeption in Angriff genommen wird. Bei einer derartigen empirischen Analyse ist dann sicherlich mit heuristisch bedingten Modifikationen des dargestellten Modells zu rechnen.

\section{LITERATUR}

Admoni, Wladimir G. (1990), Historische Syntax des Deutschen. Tübingen.

Besch, Werner / Reichmann, Oskar / Sonderegger, Stefan [Hg.] (1984/85), Sprachgeschichte. Ein Handbuch zur Geschichte der deutschen Sprache und ihrer Erforschung. 2 Bände. Berlin/New York.

Betten, Anne [Hg.] (1990), Neuere Forschungen zur historischen Syntax des Deutschen. Referate der Internationalen Fachkonferenz Eichstätt 1989. Tübingen.

Brinker, Klaus (1980), Textthematik als spezifisch textlinguistischer Forschungsbereich. in: W. Kühlwein und A. Raasch (Hg.): Sprache und Verstehen. Bd. II. Tübingen, 138-141.

Brinker, Klaus (1983), Textfunktionen. Ansätze zu ihrer Beschreibung. in: Zeitschrift für germanistische Linguistik 11, 127-148.

Buschmann, Arno (1988), Der Mainzer Reichslandfriede von 1235 und die Reichslandfrieden von Rudolf von Habsburg. in: H. Valentinitsch (Hg.), Recht und Geschichte. Graz, 105-129.

Cherubim, Dieter (1984), Sprachgeschichte im Zeichen der linguistischen Pragmatik.

in: W. Besch/ O. Reichmann/ St. Sonderegger [Hg.], Sprachgeschichte. Ein Handbuch zur Geschichte der deutschen Sprache und ihrer Erforschung. Berlin/New York, Bd. I, 802-815.

Diewald, Gabriele Maria (1991), Deixis und Textsorten im Deutschen. Tübingen.

Dijk, Teun A. van (1977), Text and Context. Explorations in the semantics and pragmatics of discourse. London/New York. 
Habermann, Mechthild (1994), Verbale Wortbildung um 1500. Eine historisch-synchrone Untersuchung anhand von Texten Albrecht Dürers, Heinrich Deichslers und Veit Dietrichs. Berlin/New York.

Halliday, M. A. K. (1978), Language as Social Semiotic. The Social Interpretation of Language and Meaning. London.

Haug, Walter und Vollmann, Benedikt K. [Hg.] (1991), Bibliothek des Mittelalters Bd.1: Frühe deutsche Literatur und lateinische Literatur in Deutschland 800-1150. Frankfurt.

Huth, Lutz (1975), Argumentationstheorie und Textanalyse. in: Der Deutschunterricht 27/6, 80-111.

Kästner, Hannes/ Schütz, Eva/ Schwitalla, Johannes (1985), Die Textsorten des Frühneuhochdeutschen. in: W. Besch/ O. Reichmann/ St. Sonderegger [Hg.], Sprachgeschichte. Ein Handbuch zur Geschichte der deutschen Sprache und ihrer Erforschung. Berlin/New York, Bd. II, 1355-1368.

Koch, Wolfgang / Rosengren, Inger / Schonebohm, M. (1981), Ein pragmatisch orientiertes Textanalyseprogramm. in: I. Rosengren (Hg.), Sprache und Pragmatik. Lunder Symposium 1980. Malmö, 155-203.

Lötscher, Andreas (1987), Text und Thema. Studien zur thematischen Konstituenz von Texten. Tübingen.

Marfurt, Bernhard (1978), Textsorten und Interaktionsmuster. in: Wirkendes Wort 30, 293-311.

Motsch, Wolfgang (1987), Zur Illokutionsstruktur von Feststellungstexten. in: Zeitschrift für Phonetik, Sprachwissenschaft und Kommunikationsforschung 40, 4567.

Motsch, Wolfgang und Viehweger, Dieter (1981), Sprachhandlung, Satz und Text. in: I. Rosengren (Hg.), Sprache und Pragmatik. Lunder Symposium 1980. Malmö, 125153.

Must, Gustav (1976), Das St. Galler Paternoster. in: Akten des V. Internationalen Germanisten Kongresses, Cambridge 1975. Jahrbuch für Internationale Germanistik 2.2, 396-403.

Müller, Rolf (1991), Ergänzende Gedanken zur Entstehungsgeschichte der Sprache, die wir Neuhochdeutsch nennen. in: J. Dittmann et al. (Hg.), Erscheinungsformen der deutschen Sprache. Berlin.

Penzl, Herbert (1975), Vom Urgermanischen zum Neuhochdeutschen. Historische Phonologie des Deutschen. Berlin.

Polenz, Peter von (1978), Geschichte der deutschen Sprache. Erw. Neubearb. der früheren Darst. von Hans Sperber. 9., überarbeitete Aufl. Berlin/New York.

- (1991), Deutsche Sprachgeschichte vom Spätmittelalter bis zur Gegenwart. Band I:

Einführung, Grundbegriffe, Deutsch in der frühbürgerlichen Zeit. Berlin/New York.

- (1994), Deutsche Sprachgeschichte vom Spätmittelalter bis zur Gegenwart. Band II:

17. und 18. Jahrhundert. Berlin/New York. 
Reichmann, Oskar (1984), Historische Lexikologie. in: W. Besch/ O. Reichmann/ St. Sonderegger [Hg.], Sprachgeschichte. Ein Handbuch zur Geschichte der deutschen Sprache und ihrer Erforschung. Berlin/New York, Bd. I, 440-460.

Ronneberger-Sibold, Elke (1989), Historische Phonologie und Morphologie des Deutschen. Eine kommentierte Bibliographie zur strukturellen Forschung. Tübingen.

Rosengren, Inger (1983), Die Textstruktur als Ergebnis strategischer Überlegungen des Senders. in: I. Rosengren (Hg.), Sprache und Pragmatik. Lunder Symposium 1982. Stockholm, 157-191.

- (1987), Begründungen und Folgerungen als kommunikative Handlungen. in: W. Motsch (Hg.), Satz, Text, sprachliche Handlung. Berlin, 179-197.

- (1992), Zur Illokutionsstruktur von Texten. in: Zeitschrift für Literaturwissenschaft und Linguistik 86, 9-51.

Sanders, Willy (1985), Die Textsorten des Altniederdeutschen (Altsächsischen). in: W.

Besch/ O. Reichmann/ St. Sonderegger [Hg.], Sprachgeschichte. Ein Handbuch zur Geschichte der deutschen Sprache und ihrer Erforschung. Berlin/New York, Bd. II, 1103-1109.

Sandig, Barbara (1978), Stilistik. Sprachpragmatische Grundlegung der Stilbeschreibung. Berlin/New York.

Schlieben-Lange, Brigitte (1976), Für eine historische Analyse von Sprechakten. in: H.

Weber / H. Weydt (Hg.), Sprachtheorie und Pragmatik. Akten des 10. Linguistischen Kolloquiums 1975. Tübingen, 113-119.

Schonebohm, M. (1980), Das Corpus des Projekts 'Fachsprachliche Kommunikation'. Lund.

Schwitalla, Johannes (1983), Deutsche Flugschriften 1460-1525. Textsortengeschichtliche Studien. Tübingen.

Sitta, Horst [Hg.] (1980), Ansätze zu einer pragmatischen Sprachgeschichte. Zürcher Kolloquium 1978. Tübingen.

Stachowiak, Herbert (1986), Pragmatik: ein neues Gemeinschaftswerk. in: ders. [Hg.], Pragmatik. Handbuch pragmatischen Denkens. Hamburg, Bd.I., XIX-L.

Stachowiak, Herbert [Hg.] (1986ff), Pragmatik. Handbuch pragmatischen Denkens. 4 Bände. Hamburg.

Steger, Hugo (1984), Sprachgeschichte als Geschichte der Textsorten/Texttypen und ihrer kommunikativen Bezugsbereiche. in: W. Besch/ O. Reichmann/ St. Sonderegger [Hg.], Sprachgeschichte. Ein Handbuch zur Geschichte der deutschen Sprache und ihrer Erforschung. Berlin/New York, Bd. I, 186-204.

Steger, Hugo / Deutrich, Helge / Schank, Gerd / Schütz, Eva (1974), Redekonstellation, Redekonstellationstyp, Textexemplar, Textsorte im Rahmen eines Sprachverhaltensmodells. Begründung einer Forschungshypothese. in: Gesprochene Sprache. Jb. des Institus für deutsche Sprache 1972. Düsseldorf.

Sperber, Hans (1926), Geschichte der deutschen Sprache. Berlin/Leipzig.

Toulmin, Stephen (1975), Der Gebrauch von Argumenten (<The Uses of Argument $>$ ). Übersetzung aus dem Engl. (Cambridge 1958), 4. Aufl. Kronberg/Ts. 
Wagner, Andreas (1994), Wie sich Sprechakte historisch verändern. Vorstudien zu einer Typologie des historischen Wandels von Sprechakten am Beispiel von SEGNEN im Althebräischen und BEKENNEN im Deutschen. in: D. W. Hallwachs und I. Stiitz (Hg.), Sprache - Sprechen - Handeln. Akten des 28. Linguistischen Kolloquiums 1993. Tübingen, 181-187.

Wichter, Sigurd (1994), Experten- und Laienwortschätze. Umriß einer Lexikologie der Vertikalität. Tübingen.

Wolf, Dieter (1984), Prinzipien und Methoden historischer Lexikologie. in: W. Besch/ O. Reichmann/ St. Sonderegger [Hg.], Sprachgeschichte. Ein Handbuch zur Geschichte der deutschen Sprache und ihrer Erforschung. Berlin/New York, Bd. I, 546-557.

\section{Zusammenfassung}

Die gegenwärtige germanistische Historiolinguistik ist geprägt durch eine pragmatische Ausweitung ihres traditionell sprachstrukturellen Erkenntnisinteresses. Allerdings stehen den programmatischen Forderungen nach einer Textorientierung sprachgeschichlicher Fragestellungen bisher nur wenige konkrete Textanalysen gegenüber. Der Beitrag nennt in der Form von Thesen drei Aspekte als Gründe für diesen Praxis-Theorie-Bruch. Die Gründe werden herausgearbeitet. Den Schwerpunkt bildet die Auseinandersetzung zur These (2), d.h. mit dem Desiderat eines textlinguistischen Konzepts zur Analyse historischer Texte. Diesbezüglich werden prinzipielle Fragestellungen zur Texttypologie und Textsortenklassifikation angesprochen, und es wird in wesentlichen Grundzügen das Konzept eines kommunikationsorientierten Verfahrens zur Analyse historischer Texte vorgestellt.

Povzetek

TIPOLOŠKE NALOGE ZGODOVINSKEGA BESEDILOSLOVJA

Sodobno germanistično zgodovinsko jezikoslovje zaznamuje pragmatično širjenje njegovega tradicionalno jezikovno strukturalnega stremljenja po spoznanju. Vsekakor se programske zahteve po uvrščanju jezikovnozgodovinskih besedil doslej lahko ponašajo le $\mathrm{z}$ redkimi konkretnimi analizami. $\mathrm{V}$ prispevku navaja avtor $\mathrm{v}$ obliki tez tri vidike, ki so vzrok razkola med teorijo in prakso. Te vzroke nato razčleni. Težišče prispevka je razprava o drugi tezi - o dezideratu besedilnojezikoslovnega koncepta za analizo zgodovinskih besedil. $V$ povezavi s tem zastavlja avtor načelna vprašanja o tipologiji besedil in klasifikaciji besedilnih vrst ter predstavi najpomembnejše značilnosti koncepta komunikativno usmerjenega postopka za analizo zgodovinskih besedil. 